Article

\title{
Body-to-Body Cooperation in Internet of Medical Things: Toward Energy Efficiency Improvement
}

\author{
Dalal Abdulmohsin Hammood ${ }^{1,2, *}$, Hasliza A. Rahim ${ }^{2}$, Ahmed Alkhayyat ${ }^{3}$ and \\ R. Badlishah Ahmad ${ }^{4}$
}

1 Electrical Engineering Technical College, Department of Computer Technical Engineering, Middle Technical University (MTU), Al Doura 10022, Baghdad, Iraq

2 Bioelectromagnetics Research Group (BioEM), School of Computer and Communication Engineering,

Universiti Malaysia Perlis (UniMAP), Kampus Pauh Putra, Arau 02600, Perlis, Malaysia;

haslizarahim@unimap.edu.my

3 Department of Computer Technical Engineering, College of Technical Engineering, The Islamic University, Najaf 54001, Iraq; ahmedalkhayyat85@gmail.com

4 School of Computer and Communication Engineering, Universiti Malaysia Perlis (UniMAP), Kampus Pauh Putra, Arau 02600, Perlis, Malaysia; badli@unimap.edu.my

* Correspondence: dalal.alsaady@eetc.mtu.edu.iq

Received: 11 September 2019; Accepted: 25 October 2019; Published: 14 November 2019

\begin{abstract}
Internet of Medical Things (IoMT) technologies provide suitability among physicians and patients because they are useful in numerous medical fields. Wireless body sensor networks (WBSNs) are one of the most crucial technologies from within the IoMT evolution of the healthcare system, whereby each patient is monitored by low-powered and lightweight sensors. When the WBSNs are integrated into IoMT networks, they are quite likely to overlap each other; thus, cooperation between WBSN sensors is possible. In this paper, we consider communication between WBSNs and beyond their communication range. Therefore, we propose inter-WBAN cooperation for the IoMT system, which is also known as inter-WBAN cooperation in an IoMT environment (IWC-IoMT). In this paper, first, a proposed architecture for the IoT health-based system is investigated. Then, a mathematical model of the outage probability for the IWC-IoMT is derived. Finally, the energy efficiency of the IWC-IoT is analysed and inspected. The simulation and numerical results show that the IWC-IoMT (cooperative IoMT) system provides superior performance compared to the non-cooperative system.
\end{abstract}

Keywords: IoMT; WBSN; inter-WBSNs cooperation; outage probability; and energy efficiency

\section{Introduction}

Currently, the Internet of Things (IoT) is one of the most powerful communication technologies of the 21st century. In the IoT environment, most of the electronic devices in our daily life will be a part of the Internet because of its communication and computing abilities. One of the important applications of IoT is healthcare. In healthcare technologies, the Internet of Medical Things (IoMT) requires several kinds of inexpensive sensors, wearable or implanted, which allow elderly people to enjoy recent advances in medical healthcare anytime, anywhere' [1-4].

Wireless body sensor network (WBSN) technologies are one of the most powerful technologies that could be utilised in the IoMT system [5]. WBSN technology comprises a group of low-power and lightweight devices with a transceiver which are used to observe the vital signs of the human body. Every sensor in WBSN is capable of gathering physiological signals, such as electrocardiogram (ECG), electroencephalogram (EEG) and movement, and sends the gathered signals to the coordinator node $(C N)$ via a wireless medium or human body channel (HBC) for further analysis and inspection [6]. 
WBSN paradigms are capable of providing long-range healthcare monitoring of humans without constraining their mobility or activity. These paradigms are used to create an intelligent and cheap healthcare-monitoring system and are used for diagnostic procedures [7].

\section{Related Work}

Despite its widespread emergence, IoMT is still in its infancy and there is a vast scope for research into various problems, such as standards, scalability, heterogeneity, common service description language, domain-specific service discovery, integration with existing IoMT systems, and so forth. In the literature, there have been a number of works in the IoT health-based paradigm, for example, a secure IoT healthcare-based paradigm that operates over the WBSN is introduced in [8]. Reliable crypto-primitives were used to build double communication techniques to guarantee transmission privacy and create entity authentication over sensors, the $C N$ and the edge of the network (server). In [9], a review of the current research in the area of WBSNs, with a specific focus on low-power consumption, transmission reliability, latency, data rates, and security is presented. In addition, the authors considered the requirements and issues of WBSN in a traditional e-healthcare paradigm, in order to discover how such paradigms are capable of efficiently communicating with the home network. An accurate statistical in-body to off-body channel model which described the signal propagation between the antenna's transceiver is provided in [10]. Where their analysis based on 3D virtual human body model. In [11], the authors considered a new structure of e-healthcare IoT-based paradigms which is referred as a secure e-healthcare IoT-based paradigm utilizing for BSN-care. In [12], an end-to-end security system for non-static e-healthcare IoT-based paradigms is proposed. In their analysis, the authors utilise the concept of a fog layer in IoT for realising seamless mobility due to fog extending the cloud system to the edge of the network. The applications of IoT in the healthcare industry are surveyed and inspected in [13], which identified the intelligentization trend of future research in e-healthcare IoT-based paradigms. An on-body sensor device with solar energy harvesting with low power transmission model which is enabled the application of an autonomous WBSN is investigated in [14]. In addition, a web-based smartphone application has been developed for showing the gathered information. In [15], the impact of power control and packet size selection over a wireless medium on the performance of e-healthcare IoT-based paradigms is considered and investigated. In their study, they propose three different protocols: a power level decision, a power level and packet size decision, and a global link decision. A novel IoT-aware Smart Hospital System is designed and studied in [16]. The proposed paradigms are capable of appropriate management of emergency conditions. Interoperability remained a significant burden for the researchers and developers of the IoT scheme. As result, in [17], an IoT-based semantic interoperability model is proposed, which provides a semantic interoperability over heterogeneous IoT devices. Physicians communicate with their patients, and the information passed between them is semantically annotated and communicated in a meaningful way. Cloud computing (CC) for IoT has emerged as a new platform in the 21st century. In [18], a new concept is established between CC and IoT: the cloud IoT-health (CC-IoT) paradigm. The term CC-IoT and several key integration challenges are considered to show a practical vision that integrates current mechanisms of CC and IoT in healthcare applications. In [19], a privacy protector is proposed, which protects the privacy of the data collected from the patient, where the Slepian-Wolf-coding-based secret sharing is utilized in Privacy Protector, which overcomes many types of security techniques. A type-2 fuzzy ontology-aided recommendation system for IoT-based healthcare to efficiently monitor the patient's body is proposed in [20]. Combining type-2 fuzzy logic and a fuzzy ontology greatly improved the prediction accuracy of a patient's situation and the accuracy rate for medicine and nutrition suggestions. Energy harvesting IoT health-based outage probability reduction, utilising inter-wireless body area network cooperation is introduced in [21]. Finally, a WBSN in an IoT health-based application, with the aim of minimizing delay and energy consumption is studied in [22]. 
The limitations of the proposed protocols in [8-22] can be elaborated as follows: (1) the cooperation between hybrid devices (such as sensors, $\mathrm{Wi}-\mathrm{Fi}$, smart devices, and so on) was not considered; (2) the cooperation between different WBSNs in IoMT was not studied; (3) finally, neither energy efficiency nor outage probability have been analysed in an IoMT environment.

The contributions from this paper are summarised as follows:

1. A new IoMT system is proposed that describes the journey of the data from the human body to the health cloud over four tiers.

2. We propose a new protocol that allows neighbouring WBSNs to cooperate with each other through different communication technologies and devices within the IoMT system. The proposed protocol is called the 'inter-WBSN cooperation in IoMT system', which is abbreviated as 'IWC-IoMT'.

3. A mathematical model of the proposed protocol is formulated and derived for two important metrics: outage probability and energy efficiency. The mathematical model describes the transmission of the data between WBSNs and beyond their communication range.

4. We reveal that the proposed IWC-IoMT protocol can achieve better performance in terms of the outage probability and energy efficiency of the IoMT system.

The rest of the paper is organised as follows: a WBSN network architecture is described in Section 2, which includes two sub-sections: WBSN in IoT-based health networks and new inter-WBSN cooperation, as well as the basic operation of the proposed protocols. In Section 3, the link analysis and outage probability of direct transmission and the proposed protocol are inspected. The energy efficiency of the different transmission scenario and the proposed protocol are investigated in Section 4. The performance and results are presented in Section 5. Finally, conclusions are drawn and directions for future work are proposed in Section 6.

\section{WBSN Networks Architecture}

\subsection{WBSN in IoMT-based Health Network}

A new architecture of an IoMT health-based paradigm is shown in the Figure 1 which can be divided into four tiers, where every layer of this proposed architecture clarified as:

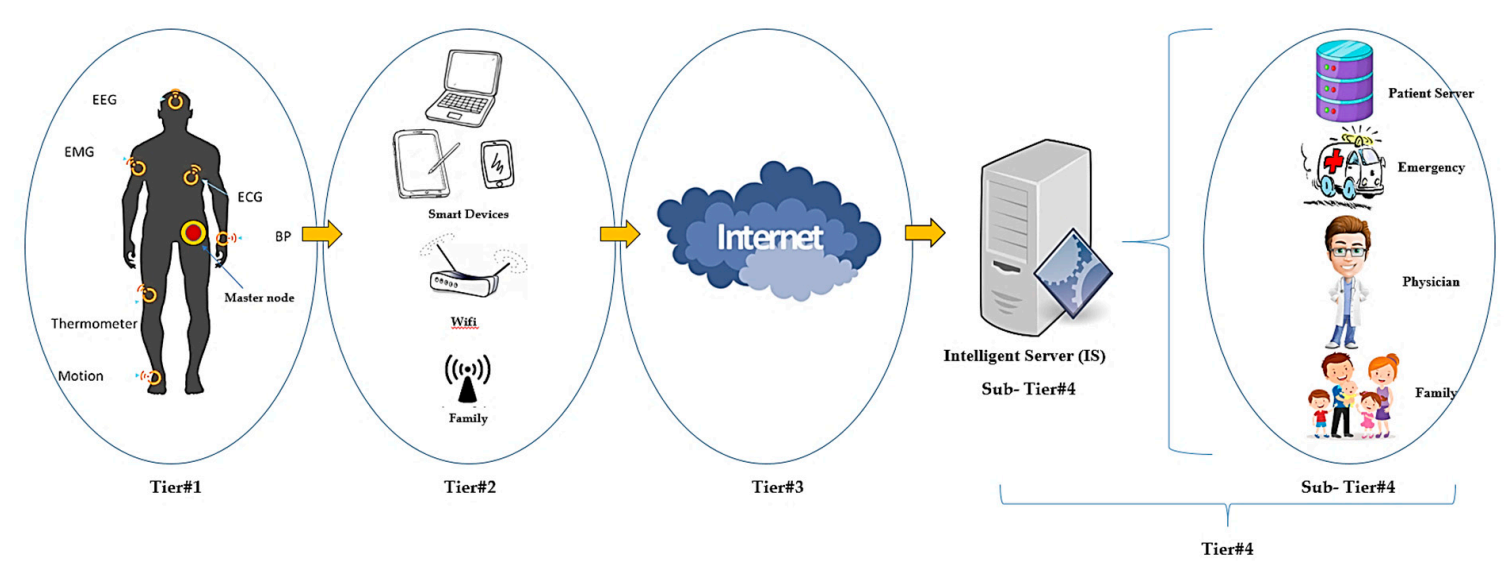

Figure 1. WBSN in IoMT-based health networks.

- The WBSN tier (tier 1): In this tier, sensors might be attached directly to the human body or sewed into fabric, or embedded inside the human body. Such sensors can be EEG, ECG, or EMG, etc. The data recorded via sensors are transmitted to the coordinator node via wireless 802.15.6 standard, after that the $C N$ transfer what were transmitted by the sensors to the next tier over one of the wireless technology or cables.

- Smart $\backslash$ wireless technology interface tier (tier 2): In this tier, smart devices are utilized, (i.e., smart phone, laptop or tablet). In this tier, data are inspected and analysed, then the data 
transferred to tier 3 over one of the smart devices or one of the chosen wireless communication technologies (i.e., Bluetooth, Wi-Fi or cellular base-station). Tier 2 represents the bridge tier that is join the WBSN to the infrastructure internet, and some time located within WBSN area.

- Infrastructure internet tier (tier 3): This level bridge the gap between the tier 2 and tier 4 via exiting backbone communication technology.

- Care-Services tier (tier 4): In this tier, the data is received and forwarded to the intelligent server (IS), the intelligent server is stored the data, analysed and forwarded to the suitable service, such as emergency response, a physician, or family.

It is clear from the above discussion that the journey of the information is entirely dependent upon received data from the first tier; if incorrect or damaged data is received from the first tier, the decision at the intelligent server might be incorrect and hazardous to the life of the patient. Therefore, we proposed a new protocol to ensure and enhance data delivery to the intelligent server.

\subsection{Proposed Inter-WBSN Cooperation Network Architecture}

In the traditional WBSN network architecture, many sensors homogeneously spread over the human body to observe any important vital signs, and the attached sensor gathers and transmits the data to the $C N$. Thus, a WBSN is based on the single-hop star topology, whereby all sensors transmit their gathered data over a wireless medium or $\mathrm{HBC}$ to the $C N$. The $C N$ then sends the data to the next tier, as explained in the previous section (shown in Figure 1).

In fact, WBSN systems are quite likely to overlap each other, and this makes the cooperation between WBAN systems possible. In this paper, we proposed a new network architecture, known as inter-WBSN cooperation in the IoMT environment. We assumed that two WBSNs were co-located in the same transmission range (area). Each WBSN consists of a single $C N$ and several sensors distributed on the human body. The operation of the proposed inter-WBSN cooperation is summarised as follows:

- Each sensor gathers the data then forwards it to the second tier (T2) over two phases, as depicted in Figure 2.

- In the first phase, sensor 1 of the WBAN1 broadcasts the gathered data to CN1 and CN2. In the second phase, $C N 1$ and $C N 2$ transmit what is received from sensor 1 to the tier 2 device (T2).

- $\quad$ The devices in $T 2$ combine the received signals via maximal ratio combing (MRC) [23].

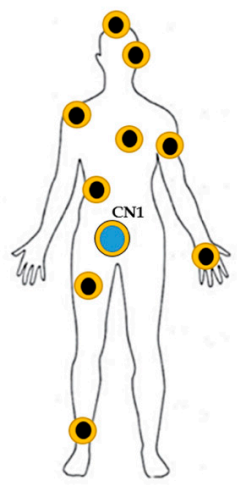

WBAN 1

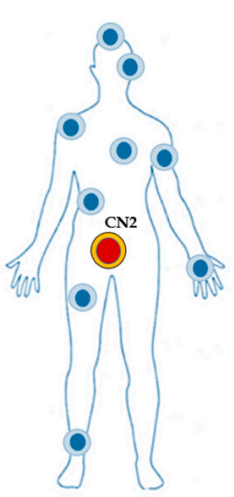

WBAN 2

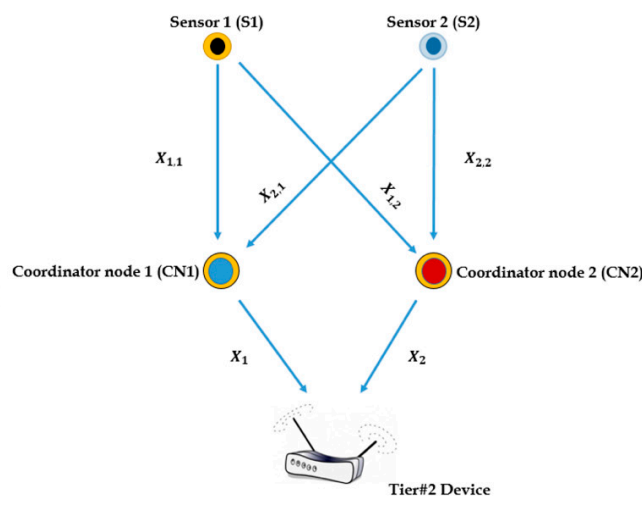

Figure 2. Network architecture of the Inter-WBSN cooperation.

In addition, we investigated two more protocols (traditional protocols): direct transmission in an IoMT environment (DT-IoMT) and two hops in an IoMT environment (TH-IoMT). In the DT-IoMT, sensor 1 transmits directly to T2; however, in the TH-IoMT, sensor 1 transmits to the $\mathrm{CN}$, and the $\mathrm{CN}$ forwards the data to $T 2$. 


\section{Link and Outage Probability Analysis}

In this section, the propagation model and the outage probability between two nodes are described. The average signal-to-noise ratio $\left(S N R_{i, j}^{a v}\right)$ from node $i$ to node $j$ is given as [24]:

$$
S N R_{i, j}^{a v}=S N R_{i, j} X_{i, j}=\frac{P_{i, j} k_{i, j}}{P_{N}+P_{I}} X_{i, j}
$$

where:

$$
k_{i, j}=\frac{G \lambda}{(4 \pi)^{2} M_{l} N_{f}}
$$

$P_{i, j}:$ is the transmission power;

$P_{N}:$ is the noise power;

$X_{i, j}$ : is a complex Gaussian random variable with unit variance;

$\left|X_{s d}\right|^{2}$ : is given as $E\left[\left|X_{i j}\right|^{2}\right]=d_{i j}^{-\alpha}$;

$d_{i j}:$ is the distance between two nodes;

$G:$ is the total gain of the transmit and receive antennae;

$\lambda:$ is the wavelength;

$N_{f}$ : is the noise figure at the receiver; and

$M_{l}:$ is the link margin.

The outage probability is defined as the probability that the transmission rate is less than or equal to the required transmission rate $\beta$. The outage probability can be calculated as [22]:

$$
P_{i, j}^{\text {out }}=P\left(\beta_{i, j} \leq \beta\right)=1-\exp \left(-U_{i, j} d_{i j}^{+\alpha_{1}}\right)
$$

where $\beta_{i, j}=\log _{2}\left(1+S N R_{i, j} X_{i, j}\right)$ and $U_{i, j}$ is expressed as:

$$
U_{i, j}=\frac{\left(2^{\beta}-1\right)}{S N R_{i, j}}
$$

\section{Outage Probability Analysis of IWC-IoMT}

The outage probability of the IWC-IoMT protocol is evaluated in this section. This analysis is based on the rate region of two-tandem transmission, as described in Figure 3, where the end-to-end outage probability of the sensor 1 to the $T 2$ link is evaluated. The rate region of the two sensors and the two CNs is shown in Figure 3. Note that the $\left(\beta_{1}, \beta_{1}\right)$ in Figure 3 is separated into four regions. We can describe the error and correct reception regions as follows: let us consider the error region of sensor 1, where in Figure 3, region 3 (R3) and region 4 (R4) corresponding to data received from sensor 1 is erroneous; while the data received from sensor 2 can be error-free in R3. In the same way, region 2 (R2) and region 4 (R4) corresponding to data received from sensor 2 is erroneous; while the data received from sensor 1 can be error-free in R2. On the other hand, region 1 (R1) corresponding to data received from sensor 1 and sensor 2 is correct.

The communication model of the IWC-IoMT, shown in Figure 2, is a two-hop communication, where the first phase is the cooperative mode and the second phase is two non-tandems (transmission 
over two different time slots) direct transmission mode. Consequently, the outage probability of the IWC-IoMT can be expressed as:

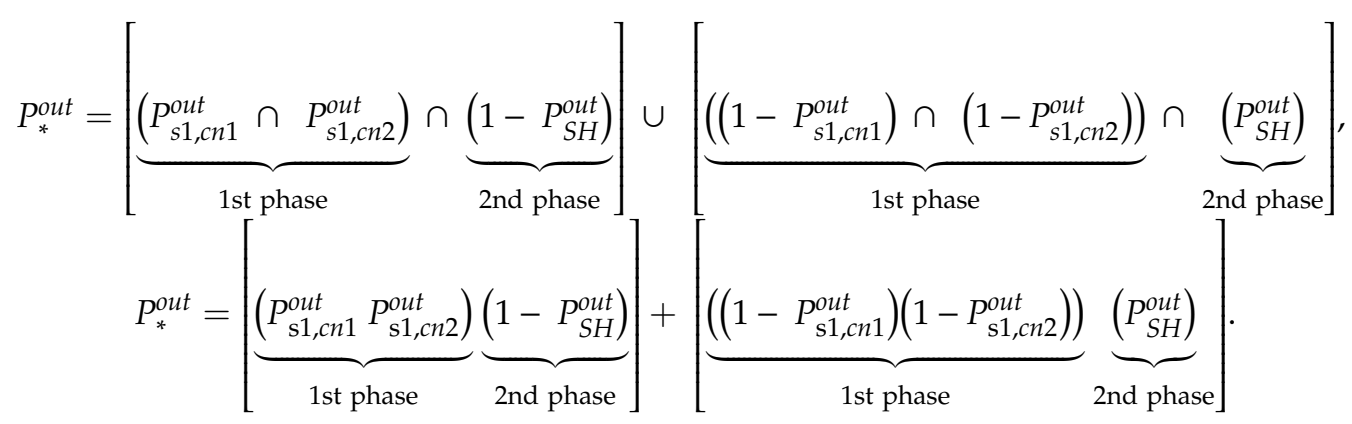

In Equation (5), the first term corresponds with the event when the first phase is in the outage, while the second phase is not in the outage and the second term corresponds to the event, which is contrast to the first event. $P_{s 1, c n 1}^{o u t}$ is the outage probability of the $S 1-C N 1$ link ( $X_{1,1}$ link) and can be expressed as:

$$
P_{s 1, c n 1}^{\text {out }}=P\left(R_{3}^{c n 1}\right)+P\left(R_{4}^{c n 1}\right)
$$

where $P\left(R_{3}^{c n 1}\right)$ and $P\left(R_{4}^{c n 1}\right)$ are the rate regions of sensor 1 to $C N 1$ (first phase). The terms $P\left(R_{3}^{c n 1}\right)$ and $P\left(R_{4}^{c n 1}\right)$ in Equation (6) can be further expressed as:

$$
\begin{gathered}
P\left(R_{3}^{c n 1}\right)=P\left[\log _{2}\left(1+S N R X_{1,1}\right)<\beta_{1}, \log _{2}\left(1+\frac{S N R X_{2,1}}{1+S N R X_{1,1}}\right)>\beta_{2}\right], \\
P\left(R_{3}^{c n 1}\right)=\int_{X_{1,1=0}}^{U_{1,1}} \exp \left(-X_{1,1}\right) d X_{1,1} \int_{X_{2,1}=U_{2,1}+X_{1,1}\left(2^{\beta_{2}}-1\right)}^{\infty} \exp \left(-X_{2,1}\right) d X_{2,1}, \\
P\left(R_{3}^{c n 1}\right)=\frac{1}{2^{\beta_{2}}}\left[\exp \left(-U_{2,1}\right)-\exp \left(-\frac{2^{\beta_{1}+\beta_{2}-1}}{S N R}\right)\right] .
\end{gathered}
$$

and:

$$
\begin{gathered}
P\left(R_{4}^{c n 1}\right)=P\left[\log _{2}\left(1+\frac{S N R X_{1,1}}{1+S N R X_{1,2}}\right)<\beta_{1}, \log _{2}\left(1+\frac{S N R X_{2,1}}{1+S N R X_{1,1}}\right)\right. \\
\left.\beta_{2}, \log _{2}\left(1+S N R X_{1,1}+S N R X_{2,1}\right)<\beta_{1}+\beta_{2}\right], \\
P\left(R_{4}^{c n 1}\right)=\int_{0}^{U_{1,1} U_{2,1}\left(1+S N R X_{1,1}\right)} \int_{0}^{U_{1,1} 2^{\beta_{2}}} \exp \left(-X_{1,1}-X_{2,1}\right) d X_{1,1} d X_{2,1} \\
+\int_{U_{1,1}} \exp \left(-X_{1,1}\right) d X_{1,1} \frac{2^{\beta_{1}+\beta_{2-1}}}{S N R}-X_{1,1} \exp \left(-X_{2,1}\right) d X_{2,1}, \\
P\left(R_{4}^{c n 1}\right)=1-\left(\frac{1}{2^{\beta 1}} \exp \left(-U_{1,1}\right)+\frac{1}{2^{\beta 2}} \exp \left(-U_{2,1}\right)\right. \\
\left.\quad+\exp \left(-\frac{2^{\beta_{1}+\beta_{2}-1}}{S N R}\right)\left(\left[1-\frac{1}{2^{\beta 1}}-\frac{1}{2^{\beta 2}}\right]+U_{1,1} U_{2,1} S N R\right)\right)
\end{gathered}
$$

Inserting Equations (8) and (7) into Equation (6), we obtain $P_{s 1, c n 1}^{\text {out }}$. In what follows, $P_{s 1, c n 2}^{\text {out }}$ is the outage probability of the $S 1-C N 2$ link ( $X_{1,2}$ link), and can be derived by similar calculations shown in Equations (6)-(8). P $P_{s 1, c n 2}^{\text {out } 2}$ is expressed as:

$$
P_{s 1, c n 2}^{\text {out }}=P\left(R_{2}^{c n 2}\right)+P\left(R_{4}^{c n 2}\right)
$$

The term $P\left(R_{2}^{c n 2}\right)$ and $P\left(R_{4}^{c n 2}\right)$ in Equation (6) can be further expressed as:

$$
P\left(R_{2}^{c n 2}\right)=\frac{1}{2^{\beta_{2}}}\left[\exp \left(-U_{2,1}\right)-\exp \left(-\frac{2^{\beta_{1}+\beta_{2}}-1}{S N R}\right)\right]
$$


and:

$$
\begin{aligned}
P\left(R_{4}^{c n 2}\right)=1- & \left(\frac{1}{2^{\beta 1}} \exp \left(-U_{1,1}\right)+\frac{1}{2^{\beta 2}} \exp \left(-U_{2,1}\right)\right. \\
& \left.+\exp \left(-\frac{2^{\beta 1}+\beta_{2}-1}{S N R}\right)\left(\left[1-\frac{1}{2^{\beta 1}}-\frac{1}{2^{\beta 2}}\right]+U_{1,1} U_{2,1} S N R\right)\right)
\end{aligned}
$$

Inserting Equations (10) and (11) into Equation (9), we obtain $P_{s 1, c n 2}^{\text {out }} . P_{S H}^{o u t}$ is the outage probability of the second phase from $C N 1$ and $C N 2$ to $T 2$. The second phase represents the transmission from the $\mathrm{CN}$ nodes to $\mathrm{T} 2$ at different time slots, and $\mathrm{T} 2$ combines the received data via MRC. Thus, the outage occurs whenever:

$$
\beta\left(S N R_{1} X_{1}, S N R_{2} X_{2}\right)<\beta_{S H}
$$

We assume that each $C N$ node transmits what is received from sensor 1 over a period of time denoted as $\omega$. Thus, CN1 transmits the data to T2 via $\omega$, and $C N 2$ transmits the data to $T 2$ via $1-\omega=\bar{\omega}$, where the ratio $\omega(0<\omega<1)$. Then, Equation (12) can be further expressed as:

$$
\beta\left(S N R_{1} X_{1}, S N R_{2} X_{2}\right)=\log _{2}\left(\left(1+S N R_{1} X_{1}\right)^{\omega}\left(1+S N R_{2} X_{2}\right)^{\bar{\omega}}\right)
$$

where the outage probability $P_{S H}^{o u t}$ can then be evaluated over the outage region in Equation (13) as:

$$
\begin{gathered}
P_{S H}^{\text {out }}=P\left[\left(1+S N R_{1} X_{1}\right)^{\omega}\left(1+S N R_{2} X_{2}\right)^{\bar{\omega}}<\beta_{S H}\right], \\
P_{S H}^{\text {out }}=\int_{0}^{U_{S H 1}} \exp \left(-X_{1}\right) d X_{1} \int_{0}^{U_{S H 2}} \exp \left(-X_{2}\right) d X_{2}, \\
P_{S H}^{\text {out }}=\left(1-\exp \left(-U_{S H 1}\right)\right)\left(1-\exp \left(-U_{S H 2}\right)\right) .
\end{gathered}
$$

where $U_{S H 1}$ and $U_{S H 2}$ can then be expressed as:

$$
\begin{aligned}
& U_{S H 1}=\frac{\left(2^{\beta S H}-1\right)^{\omega}}{S N R_{1}}, \\
& U_{S H 1}=\frac{\left(2^{\beta}-1\right)^{\bar{\omega}}}{S N R_{2}} .
\end{aligned}
$$

where $U_{S H 1}$ and $U_{S H 2}$ represent $C N 1-T 2$ link and $C N 2-T 2$ link, respectively.

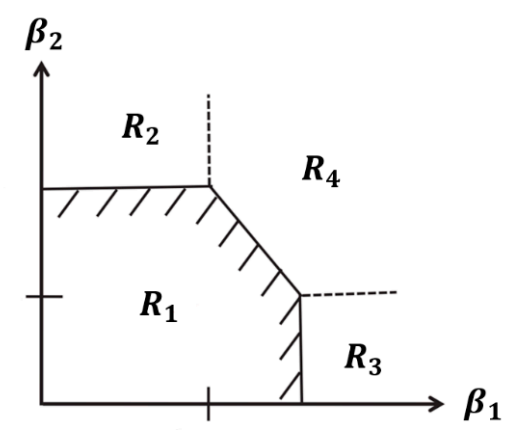

Figure 3. Rate region of IWC-IoMT protocol, where $\beta_{1}=\beta_{s 1, c n 1}$, and $\beta_{2}=\beta_{s 1, c n 2}$.

\section{Energy Efficiency of the IWC-IoMT}

Energy efficiency is denoted as the achievable information transmission per joule of energy consumption. In this section, the energy efficiencies of DT-IoMT, TH-IoMT and IWC-IoMT are derived based on the previous calculations. The energy efficiency between two nodes or the energy efficiency of the DT-IoMT is defined as [25]:

$$
E_{i, j}=\left(1-P_{i, j}^{o u t}\right) \frac{\beta_{i, j}}{P_{i, j}^{t o t}} \quad[\mathrm{bit} / \text { joule }]
$$


where $P_{i, j}^{\text {tot }}$ is the total power consumption and is comprised of $P_{a m p}, P_{t x}$ and $P_{r x}$, the $P_{a m p}$ is the power amplifier consumption for the transmission, and $P_{t x}$ and $P_{r x}$ are the power consumption of the internal circuitry for transmitting and receiving, respectively. $P_{i, j}^{\text {tot }}$ is expressed as:

$$
P_{i, j}^{t o t}=P_{a m p}+P_{t x}+P_{r x}
$$

In what follows, the energy efficiency of the TH-IoMT, $E_{T H}$ is expressed as:

$$
E_{T H}=\frac{\left(1-P_{s 1, c n 1}^{o u t}\right)\left(1-P_{c n 1, T 2}^{o u t}\right) \beta_{T H}}{P_{T H}^{\text {tot }}}
$$

where in the two-hop cooperation, two nodes are involved in the data transmission and the total power consumption, $P_{T H}^{\text {tot }}$, and the achievable link capacity is equal to the minimum of the two hops, $\beta_{T H}$, which are expressed as:

$$
\begin{gathered}
P_{T H}^{t o t}=2\left(P_{a m p}+P_{t x}+P_{r x}\right) \\
\beta_{T H} \approx \beta_{s 1, c n 1} \approx \beta_{c n 1, T 2}
\end{gathered}
$$

When we insert Equations (19) and (20) into Equation (18), we obtain $E_{T H}$. Next, the energy efficiency of the IWC-IoMT protocol for sensor 1 is expressed as:

$$
E_{*}=\frac{\left(1-P_{s 1, c n 1}^{\text {out }}\right)\left(1-P_{S 1, c n 2}^{\text {out }}\right) \beta_{F H}}{P_{a m p}+P_{t x}+2 P_{r x}}+\frac{\left(1-P_{c n 1, T 2}^{\text {out }}\right)\left(1-P_{c n 2, T 2}^{\text {out }}\right) \beta_{S H}}{2 P_{a m p}+2 P_{t x}+P_{r x}}
$$

where $\left(1-P_{s 1, c n 1}^{\text {out }}\right)\left(1-P_{c n 1, T 2}^{\text {out }}\right)$ represent the successful transmission probability from $S 1$ to $C N 1$, and from $S 1$ to $C N 2$, respectively, and $\left(1-P_{c n 1, T 2}^{\text {out }}\right)\left(1-P_{c n 2, T 2}^{\text {out }}\right)$ represent the successful transmission probability from $C N 1$ to $T 2$, and from $C N 2$ to $T 2$, respectively. The first term total power, $P_{a m p}+P_{t x}+2 P_{r x}$, two nodes are involved in the data reception and a single node is involved in data transmission. Then, the second term total power, $2 P_{a m p}+2 P_{t x}+P_{r x}$, two nodes are involved in the data transmission and a single node is involved in data reception. Furthermore, the achievable link capacity of the first and second terms is expressed as:

$$
\begin{aligned}
& \beta_{F F}=\beta_{s 1, c n 1}=\beta_{s 1, c n 2} \\
& \beta_{S F}=\beta_{c n 1, T 2}=\beta_{c n 2, T 2}
\end{aligned}
$$

where $\beta_{F F}$ is the transmission rate from the sensors to the coordinators (first phase), and $\beta_{S F}$ is the transmission rate from the coordinators to $T 2$ (second hop). Finally, if we insert Equations (21) and (22) into Equation (20), we obtain $E_{*}$.

\section{Simulation and Discussion}

In this section, we evaluate the performance of the proposed inter-WABN cooperation of IoMT health-based systems via computer simulations. In the simulations, random topology, various wireless body sensors are located in a $3 \times 3 \mathrm{~m}$, and two human bodies are assumed to coexist in the same range. The distances are assumed to be variable in the simulations, and all links are assumed to have the same distance which is denoted as $d_{0}$. The transmission rate of all the links is assumed to be $\beta_{o}(\mathrm{~b} / \mathrm{s} / \mathrm{Hz})$. The path-loss exponent, $\alpha$, is $4, M l=40 \mathrm{~dB}$ and $N f=10 \mathrm{~dB}$, the total antenna gain is $G=5 \mathrm{dBi}$, the carrier frequency is $f_{C}=2.5 \mathrm{GHz}$, and $N_{o}=-70 \mathrm{dBm}$. In what follows, we denote the power consumption of circuitry for amplifying, transmitting and receiving as $P_{o}(\mathrm{~mW})$.

Figure 4 shows the comparison of outage probabilities of DT-IoMT, TH-IoMT and IWC-IoMT as a function of the inter-node distance, $d_{0}$. In general, the outage probabilities of all communication reduced as the inter-nodes distance increases. As shown in the figure, the proposed protocol achieved 
better performance compared to both protocols. As expected, the performance of the TH-IoMT is better compared to the DT-IoMT because the data are transmitted over two independent links.

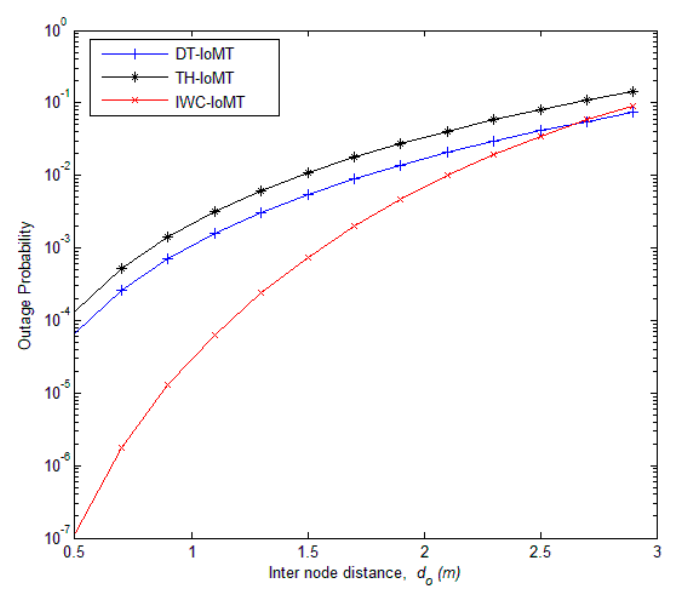

Figure 4. Outage probability versus internode distance, $d_{0}$. For all cases and links, the transmission rate and power consumption, $P_{o}$, are $2 \mathrm{~b} / \mathrm{s} / \mathrm{Hz}$ and $10 \mathrm{dBm}$.

Figures 5 and 6 show the comparison of outage probabilities of DT-IoMT, TH-IoMT and IWC-IoMT as a function of the inter-node distance, $d_{0}$. In both figures, an asymmetric transmission rate is assumed. In Figure 5 and for the first phase (i.e., from the sensors to the $C N$ ) is set to $5 \mathrm{~b} / \mathrm{s} / \mathrm{Hz}$ and for the second phase (i.e., from the $\mathrm{CN}$ to $T 2$ ) is set to $2 \mathrm{~b} / \mathrm{s} / \mathrm{Hz}$. While, the transmission rates of the DT-IoMT and TH-IoMT are set to $5 \mathrm{~b} / \mathrm{s} / \mathrm{Hz}$. As shown in the figure, the performance of IWC-IoMT is outperformed the DT-IoMT and TH-IoM protocols at distance less than $1.5 \mathrm{~m}$, while the performance of the IWC-IoMT is degraded at distance more than $1.5 \mathrm{~m}$. In the Figure 6 and for the first phase (i.e., from the sensors to the $C N$ ) is set to $2 \mathrm{~b} / \mathrm{s} / \mathrm{Hz}$ and for the second phase (i.e., from the $C N$ to $T 2$ ) is set to $5 \mathrm{~b} / \mathrm{s} / \mathrm{Hz}$. While the transmission rate of the DT, THC and ICC is set to $5 \mathrm{~b} / \mathrm{s} / \mathrm{Hz}$. As shown in the figure, the performance of the IWC-IoMT is less than DT-IoMT and TH-IoMT for all cases and the inter-nodes distance.

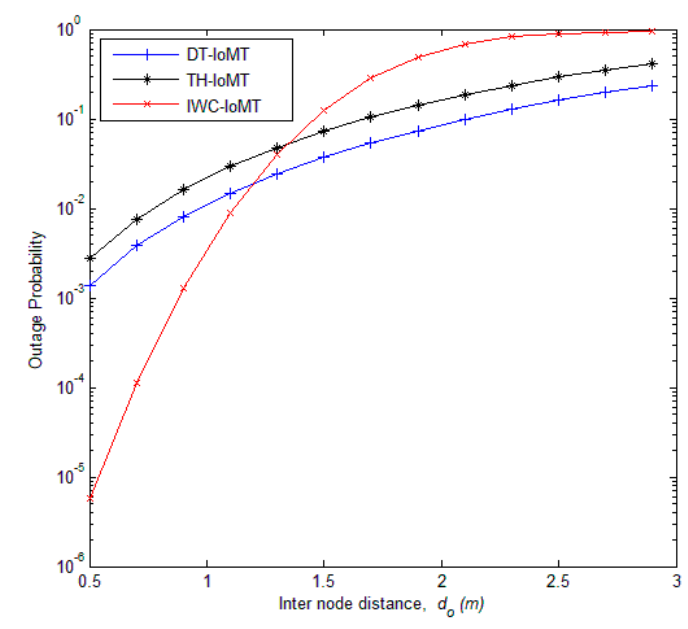

Figure 5. Outage probability versus internode distance, $d_{0}$. For all cases and links, power consumption, $P_{o}$, is $10 \mathrm{dBm}$.

Figure 7 shows the comparison of energy efficiencies of DT-IoMT, TH-IoMT and IWC-IoMT as a function of the inter-node distance, $d_{0}$. As shown in Figure 7 , the energy efficiency values of all protocols are decreased as the inter-node distance increased. This performance of all protocols decayed due to the greater transmission power required to guarantee a certain transmission rate. 
Nevertheless, IWC-IoMT performs best among all protocols because the IWC-IoMT utilised four independent transmission links and MRC at the destination (T2).

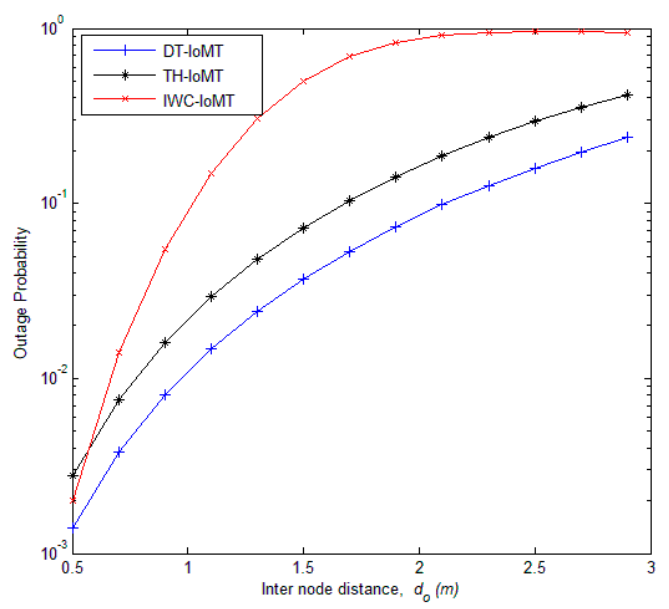

Figure 6. Outage probability versus internode distance, $d_{0}$. For all cases and links, power consumption, $P_{o}$, is $10 \mathrm{dBm}$.

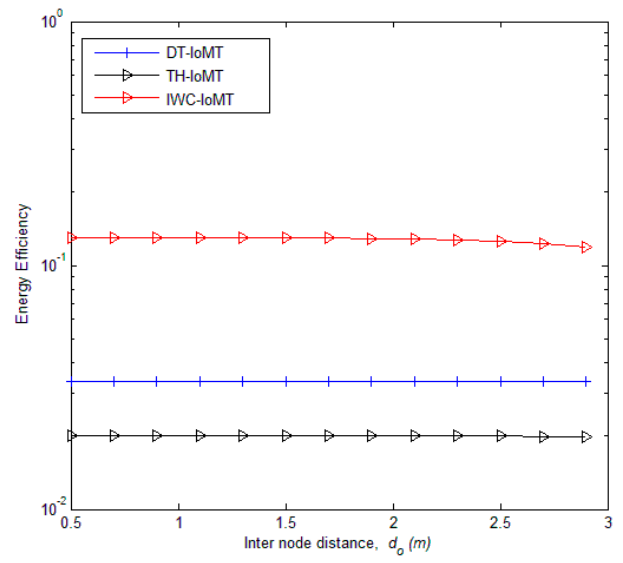

Figure 7. Energy efficiency versus internode distance, $d_{0}$. For all cases and links, power consumption, $P_{0}$, is $10 \mathrm{dBm}$.

Figure 8 shows the comparison of energy efficiencies of DT-IoMT, TH-IoMT and IWC-IoMT as a function of the power consumption, $P_{o}$. From Figure 8, we can see that the energy efficiencies of DT-IoMT, TH-IoMT and IWC-IoMT increase as the power consumption increases. The reason is that the energy efficiencies are inversely proportional to the power consumption. In comparison, IWC-IoMT has greater energy efficiency compared to the DT-IoMT and TH-IoMT because its data is transmitted over four independent paths and the T2 utilized MRC. The energy efficiency of the DT-IoMT is generally less than that of the TH-IoMT. The reason is that the data is transmitted over two independent paths in the TH-IoMT.

Figure 9 shows the comparison of energy efficiencies of DT-IoMT, TH-IoMT and IWC-IoMT as a function of the power consumption, $\beta_{0}(\mathrm{~b} / \mathrm{s} / \mathrm{Hz})$. We can see that the energy efficiencies of all protocols are decreased with the increase in the transmission rate. The reason is that, with the increase in the transmission rate, the nodes or the sensors need more power to achieve the required transmission rate. As expected, the performance of the IWC-IoMT outperforms the performance of the DT-IoMT and TH-IoMT, however, the performance of the TH-IoMT is better than the DT-IoMT performance. 


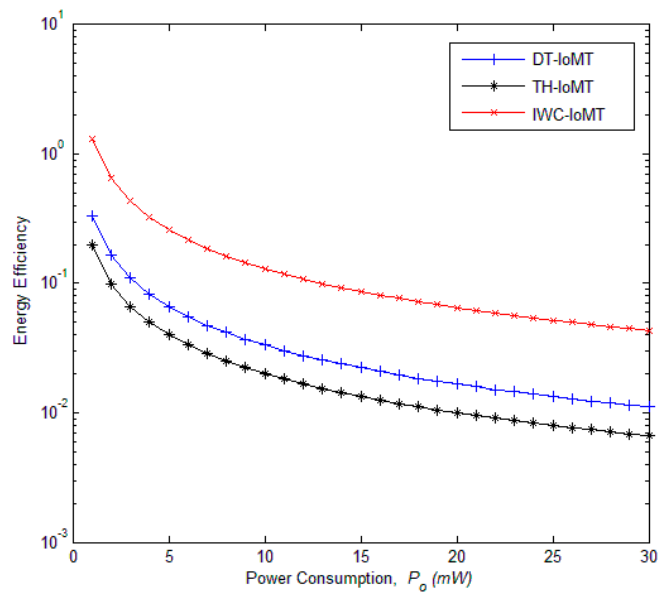

Figure 8. Energy efficiency versus power consumption, $P_{o}$. For all cases and links, the transmission rate is $2 \mathrm{~b} / \mathrm{s} / \mathrm{Hz}$.

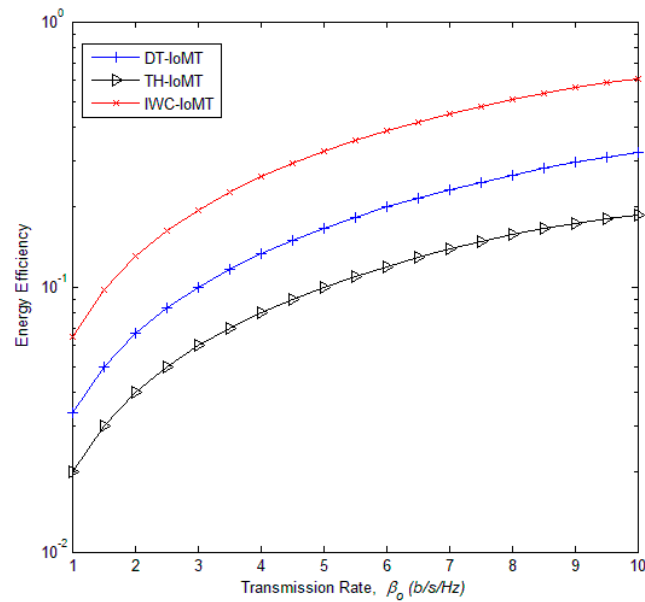

Figure 9. Energy efficiency versus transmission rate, $\beta_{0}$. For all cases and links, power consumption, $P_{O}$, is $10 \mathrm{dBm}$.

\section{Conclusions}

Recent advances in the design of Internet of Medical Things (IoMT) technologies are encouraging the development of smart systems to support and improve healthcare. In this paper, we have designed a new system of the IoMT. The proposed IoMT consists of four tiers, starting from human body sensors and ending with the health cloud. Along with the proposed design, we proposed a new protocol known as IWC-IoMT. The proposed protocol would allow two bodies to cooperate with each other to deliver data efficiently.

In this work, two important metrics were considered: outage probability and energy efficiency. The results show that the proposed protocol achieved better performance when compared to non-inter-WBAN cooperation for a symmetric transmission rate. On the other hand, the performance of the proposed protocol degraded when the asymmetric transmission rates were utilised.

In future work, we will analyse the proposed protocol with an energy harvesting technique that allows the coordinator nodes to first harvest the energy and then cooperate based on a time switch.

Author Contributions: Formal analysis: H.A.R.; funding acquisition: A.A.; methodology: A.A.; resources: D.A.H.; supervision: R.B.A.; validation: D.A.H.; writing—original draft: A.A.; writing—review and editing: H.A.R.

Conflicts of Interest: The authors declare no conflict of interest. 


\section{References}

1. Yang, G.; Xie, L.; Mäntysalo, M.; Zhou, X.; Pang, Z.; Da Xu, L.; Kao-Walter, S.; Chen, Q.; Zheng, L.R. A health-IoT platform based on the integration of intelligent packaging, unobtrusive bio-sensor, and intelligent medicine box. IEEE Trans. Ind. Inform. 2014, 10, 2180-2191. [CrossRef]

2. Hassanalieragh, M.; Page, A.; Soyata, T.; Sharma, G.; Aktas, M.; Mateos, G.; Kantarci, B.; Andreescu, S. Health monitoring and management using Internet-of-Things (IoT) sensing with cloud-based processing: Opportunities and challenges. In Proceedings of the 2015 IEEE International Conference on Services Computing 2015, New York, NY, USA, 27 Jun-2 July 2015; IEEE: Piscataway, NJ, USA, 2015; pp. $285-292$.

3. Bui, N.; Zorzi, M. Health care applications: A solution based on the internet of things. In Proceedings of the 4th International Symposium on Applied Sciences in Biomedical and Communication Technologies, Barcelona, Spain, 26-29 October 2011; ACM: New York, NY, USA, 2011; p. 131.

4. Santos, J.; Rodrigues, J.J.; Silva, B.M.; Casal, J.; Saleem, K.; Denisov, V. An IoT-based mobile gateway for intelligent personal assistants on mobile health environments. J. Netw. Comput. Appl. 2016, 71, $194-204$. [CrossRef]

5. Kumar, P.; Lee, H.J. Security issues in healthcare applications using wireless medical sensor networks: A survey. Sensors 2012, 12, 55-91. [CrossRef] [PubMed]

6. Al-Mishmish, H.; Alkhayyat, A.; Rahim, H.; Hammood, D.; Ahmad, R.; Abbasi, Q. Critical data-based incremental cooperative communication for wireless body area network. Sensors 2018, 18, 3661. [CrossRef] [PubMed]

7. Abdulmohsin Hammood, D.; Rahim, H.A.; Alkhayyat, A.; Ahmed, R.B.; Abbasi, Q.H. Reliable emergency data transmission using transmission mode selection in wireless body area network. Cogent Eng. 2018, 5, 1562859. [CrossRef]

8. Yeh, K.H. A secure IoT-based healthcare system with body sensor networks. IEEE Access 2016, 4, 10288-10299. [CrossRef]

9. Ghamari, M.; Janko, B.; Sherratt, R.S.; Harwin, W.; Piechockic, R.; Soltanpur, C. A survey on wireless body area networks for ehealthcare systems in residential environments. Sensors 2016, 16, 831. [CrossRef]

10. Liao, Y.; Leeson, M.; Higgins, M.; Bai, C. Analysis of in-to-out wireless body area network systems: Towards QoS-aware health internet of things applications. Electronics 2016, 5, 38. [CrossRef]

11. Madhumathi, R.M.; Jagadeesan, A.; Kaushik, S. Healthcare Monitoring System Using Body Sensor Network. In Proceedings of the International Conference on Engineering Innovations and Solutions (ICEIS-2016), Rome, Italy, 25-28 April 2016; pp. 171-176.

12. Moosavi, S.R.; Gia, T.N.; Nigussie, E.; Rahmani, A.M.; Virtanen, S.; Tenhunen, H.; Isoaho, J. End-to-end security scheme for mobility enabled healthcare Internet of Things. Future Gener. Comput. Syst. 2016, 64, 108-124. [CrossRef]

13. Yuehong, Y.I.; Zeng, Y.; Chen, X.; Fan, Y. The internet of things in healthcare: An overview. J. Ind. Inform. Integr. 2016, 1, 3-13.

14. Wu, T.; Wu, F.; Redouté, J.M.; Yuce, M.R. An autonomous wireless body area network implementation towards IoT connected healthcare applications. IEEE Access 2017, 5, 11413-11422. [CrossRef]

15. Catarinucci, L.; De Donno, D.; Mainetti, L.; Palano, L.; Patrono, L.; Stefanizzi, M.L.; Tarricone, L. An IoT-aware architecture for smart healthcare systems. IEEE Internet Things J. 2015, 2, 515-526. [CrossRef]

16. Chen, X.; Ma, M.; Liu, A. Dynamic power management and adaptive packet size selection for IoT in e-Healthcare. Comput. Electr. Eng. 2018, 65, 357-375. [CrossRef]

17. Jabbar, S.; Ullah, F.; Khalid, S.; Khan, M.; Han, K. Semantic interoperability in heterogeneous IoT infrastructure for healthcare. Wireless Commun. Mobile Comput. 2017, 2017. [CrossRef]

18. Darwish, A.; Hassanien, A.E.; Elhoseny, M.; Sangaiah, A.K.; Muhammad, K. The impact of the hybrid platform of internet of things and cloud computing on healthcare systems: Opportunities, challenges, and open problems. J. Ambient Intell. Humaniz. Comput. 2019, 10, 4151-4166. [CrossRef]

19. Luo, E.; Bhuiyan, M.Z.; Wang, G.; Rahman, M.A.; Wu, J.; Atiquzzaman, M. Privacyprotector: Privacy-protected patient data collection in IoT-based healthcare systems. IEEE Commun. Mag. 2018, 56, 163-168. [CrossRef]

20. Ali, F.; Islam, S.R.; Kwak, D.; Khan, P.; Ullah, N.; Yoo, S.J.; Kwak, K.S. Type-2 fuzzy ontology-aided recommendation systems for IoT-based healthcare. Comput. Commun. 2018, 119, 138-155. [CrossRef] 
21. Thabit, A.A.; Mahmoud, M.S.; Alkhayyat, A.; Abbasi, Q.H. Energy harvesting Internet of Things health-based paradigm: Towards outage probability reduction through inter-wireless body area network cooperation. Int. J. Distrib. Sens. Netw. 2019, 15, 10. [CrossRef]

22. Alkhayyat, A.; Thabit, A.A.; Al-Mayali, F.A.; Abbasi, Q.H. WBSN in IoT Health-Based Application: Toward Delay and Energy Consumption Minimization. J. Sens. 2019, 2019. [CrossRef]

23. Hammood, D.A.; Rahim, H.A.; Badlishah Ahmad, R.; Alkhayyat, A.; Salleh, M.E.M.; Abdulmalek, M.; Jusoh, M.; Abbasi, Q.H. Enhancement of the Duty Cycle Cooperative Medium Access Control for Wireless Body Area Networks. IEEE Access 2019, 7, 3348-3359. [CrossRef]

24. Laneman, J.N.; Tse, D.N.C.; Wornell, G.W. Cooperative diversity in wireless networks: Efficient protocols and outage behavior. IEEE Trans. Inform. Theory 2004, 50, 3062-3080. [CrossRef]

25. Promwongsa, N.; Sanguankotchakorn, T. Packet size optimization for energy-efficient 2-hop in multipath fading for WBAN. In Proceedings of the 2016 22nd Asia-Pacific Conference on Communications (APCC), Yogyakarta, Indonesia, 25-27 August 2016; IEEE: Piscataway, NJ, USA, 2016; pp. 445-450.

(C) 2019 by the authors. Licensee MDPI, Basel, Switzerland. This article is an open access article distributed under the terms and conditions of the Creative Commons Attribution (CC BY) license (http://creativecommons.org/licenses/by/4.0/). 